\title{
Narrow-band filtering by means of triangular meta-material resonators based on RF MEMS cantilevers in CPW configuration
}

\author{
Andrea Lucibello ${ }^{1} \cdot$ Emanuela Proietti $^{1} \cdot$ Romolo Marcelli $^{1} \cdot$ Giovanni Maria Sardi $^{1}$ \\ Giancarlo Bartolucci ${ }^{2}$
}

Received: 23 July 2015 / Accepted: 4 January 2016 / Published online: 8 January 2016

(C) Springer-Verlag Berlin Heidelberg 2016

\begin{abstract}
Triangular resonators, designed by using Radio Frequency Micro Electro Mechanical Systems (RF MEMS) cantilevers in coplanar waveguide (CPW) configuration, have been proposed, fabricated on alumina and on silicon substrates and tested up to $40 \mathrm{GHz}$. They can be utilized as building blocks for realizing reconfigurable filters. A triangular split ring resonator has been etched in the central conductor of the CPW transmission line and the split is provided by the presence of a technologically actuated cantilever beam, i.e. with metal directly deposited without the sacrificial layer, to emulate the ideal actuation and to get preliminary information of the expected microwave response in comparison to numerical simulations. Two main configurations of the triangular transmission line have been studied for narrow-band microwave switching, the first one without the MEMS cantilever and the second one with a technologically actuated MEMS cantilever switch. Because of the contributions of both the resonator and the switch, the device has a narrow band filtering feature. Moreover, these geometries can also be characterized in the broad class of metamaterial devices, because the unit cell can be described by negative equivalent electric permittivity and/or magnetic permeability, by means of a transmission line parameters extraction method. This finding contributes to the possibility of miniaturization implementations of the resonating structures. A simple but effective
\end{abstract}

Romolo Marcelli

Romolo.Marcelli@imm.cnr.it

1 National Research Council, Institute for Microelectronics and Microsystems, Via del Fosso del Cavaliere 100, 00133 Rome, Italy

2 Department of Electronic Engineering, University of Roma Tor Vergata, Via del Politecnico 1, 00133 Rome, Italy lumped element circuit for the modeling of the single triangular resonator is presented in the end.

\section{Introduction}

Most of the technological growth in the last decades in signal processing solutions at high frequencies dealt with the development of materials with novel properties. In particular, the analysis and synthesis of materials with specific electrical and magnetic properties are now a research area of high strategic interest for the foreseeable impact on a wide range of applications and technologies, such as telecommunications, information technology, aerospace, biomedical engineering and material science.

The concept of metamaterial (MM) was introduced at the end of 1960, but only recently the implementation of models has focused interest on practical applications. MMs are characterized by strong electric and magnetic plasmonic resonances that make the medium dispersive in narrow frequency ranges. In the region of resonance, the relative values of the permeability $\mu$ and of the permittivity $\varepsilon$ for MMs may be both negative. These particular artificial materials show phase and group velocity having opposite sign and a negative refractive index in certain frequency bands, characteristics that make them good candidates for applications in Radio Frequency (RF) and microwave systems.

In microwave communications, narrow band-pass filtering for both $\mathrm{X}$ - and Ku-bands is required for applications such as signal tracking, sub-band filtering or noise suppression. Generally speaking, X-band satellite service is better suited to satisfy large bandwidth requirements and is very dependable and less susceptible to weather related service outages. Conversely, Ku-band operates with a smaller satellite dish antenna and less expensive equipment, which 
makes it more attractive for small networks seeking shared bandwidth service solutions. Both of these bandwidth frequencies deliver the same speed capability, service efficiency, and they can support any advanced communications service that may be required. Ku-band transmissions do not need the large satellite antenna or the power amplification level of X-band. Ku-band also does not share the same frequency range with microwave links or other technologies like $\mathrm{X}$-band. However, $\mathrm{Ku}$ is more sensitive to heavy rain fade. Since both of them are of interest in microwave communications, we have studied two frequencies samples in these bands ( 8 and $16 \mathrm{GHz}$ ).

This paper presents the design, the fabrication and RF test of a structure realized by triangular split ring resonators, in which the split is done by cantilever MEMS switches placed along the arms of the triangle. The structure, because of the equivalent transmission line model, can also be defined a "metamaterial". Transmission characteristics of the resulting cells are obtained both numerically and experimentally. It is observed that the activation of the MEMS (the presence of the split) leads to the resonance of the device. Furthermore, an equivalent circuit composed by a shunt connected RLC cell is presented. This model can be easily implemented in any commercial software package typically used for the simulation of microwave networks.

\section{Design}

Numerous types of different ring and ring-like structures such as circular, square, $\Omega$-shaped, U-shaped, S-shaped and others have been used to create new MM transmission lines (Engheta and Ziolkowski 2006). There are few studies about the triangular shaped resonators in literature (Sabah 2008; Sabah et al. 2010; Jalali et al. 2009a, b; Vidyalakshmi and Raghavan 2009; Zhu et al. 2008, 2009), and the first study of the MMs to obtain a triangular resonator was conducted in 2008 (Sabah and Uckun 2008). In this work, new MMs comprised of triangular split ring resonators have been simulated and fabricated in coplanar waveguide configuration and a retrieval method is used for the calculation of the effective material parameters. Coplanar waveguide (CPW) designs prove to be advantageous when integration with lumped or active components is required. In addition to that, CPW technology offers low dispersion and simple realization of short-circuited ends without the need of vias, which leads to the reduction of the radiation losses and of the fabrication efforts (Wolf 2006).

The triangular shape has been not frequently debated in literature, also because of some difficulties in easily modelling it. Triangular resonators for filtering application in microstrip technology are the only similar geometry discussed (Hong and Li 2004; Xiao 2012). Anyway, looking to advantages and drawbacks of such a shape, it can be convenient with respect to classical rings for the reasons outlined in Table 1.

We used two different substrates: in a first run an alumina one (relative permittivity $\varepsilon_{\mathrm{r}} \approx 9.8$, dielectric losses $\tan \delta \approx 10^{-3}$ ) with a thickness of $635 \mu \mathrm{m}$, and in a second run a $525 \mu \mathrm{m}$ thick silicon wafer $\left(\varepsilon_{\mathrm{r}} \approx 11.7\right.$, $\tan \delta \approx 10^{-3}$ ) (Krupka et al. 2006). In both cases, we designed a triangular split ring resonator etched in the central conductor of the CPW transmission line. The split is provided by a cantilever beam (see Fig. 1), which has to be electrostatically actuated in the real case, for mimicking the behavior of the switch. In the present case, such an ideal situation was obtained for both alumina and silicon by a metal deposition with the typical shape and size of the RF MEMS switches $(200 \times 300 \mu \mathrm{m})$, even including the holes used for modulating the mechanical properties and for facilitating the sacrificial layer removal, so obtaining the switch in the "ON" state. The structure of a RF MEMS cantilever switch consists of a thin gold membrane cantilever clamped only on one side of the bridge, suspended over the etched triangle.

When the switch is in the OFF position (cantilever up) the arm of the etched triangle is not split, and the full CPW triangular line provides just a tapering for a matched transmission line from port 1 to port 2 . When the cantilever is down, i.e. the switch is actuated, the ON state provides now the split of the triangle. The Fig. 1 inset on the right upper corner gives evidence for a suspended cantilever, single side clamped, to be collapsed by means of an electrostatic actuation for closing the circuit with the inner triangle. In this way the resonance contribution coming from both the patch and the slot is evidenced by the connection of the triangular slot ring with the floating patch $(\mathrm{ON}$

Table 1 Main differences, advantages and drawbacks for resonators having circular, square and triangular shapes

\begin{tabular}{lll}
\hline Geometry & Advantages & Drawbacks \\
\hline Circle & Symmetric and free from discontinuities, easy to be modelled analytically & Planar coupling is not allowed side-by-side \\
Square & Symmetric, easy to be modelled and coupled with other square elements & Space occupancy \\
Triangle & $\begin{array}{c}\text { Axial symmetry, easy to be coupled side-by-side, } \\
\text { more compact with respect to the above geometries }\end{array}$ & $\begin{array}{c}\text { Difficult to be modeled analytically, } \\
\text { EM efforts needed to develop arrays }\end{array}$ \\
\hline
\end{tabular}




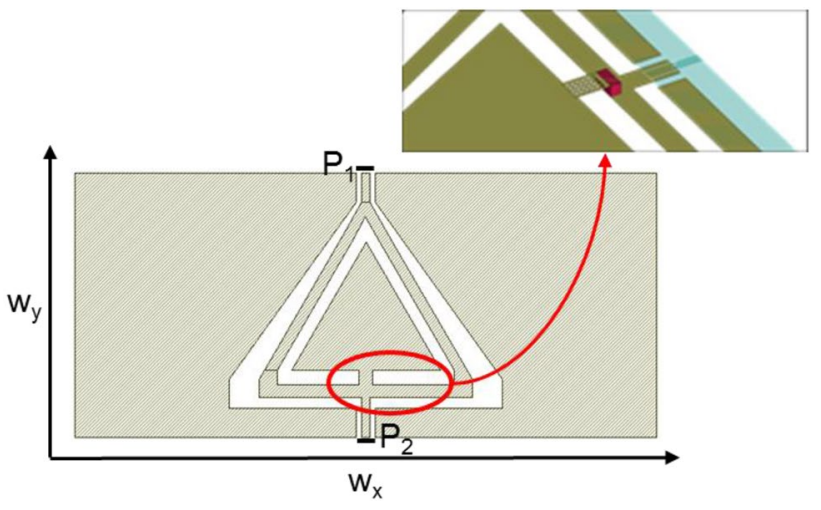

Fig. 1 Microwave Office Simulator top view of a metal gold triangular split resonator ( $\mathrm{Au}$ is grey in the figure) etched in the central conductor of the CPW transmission line (a). The gap (split) in the inner triangle has been implemented by the presence of a RF-MEMS switch. $P_{1}$ and $P_{2}$ are the input and the output port respectively. The RF MEMS switch is in the UP position in (b), corresponding to the OFF response of the resonator. When actuated, the cantilever goes in the DOWN position, connecting the inner and outer triangles and generating a slot resonance
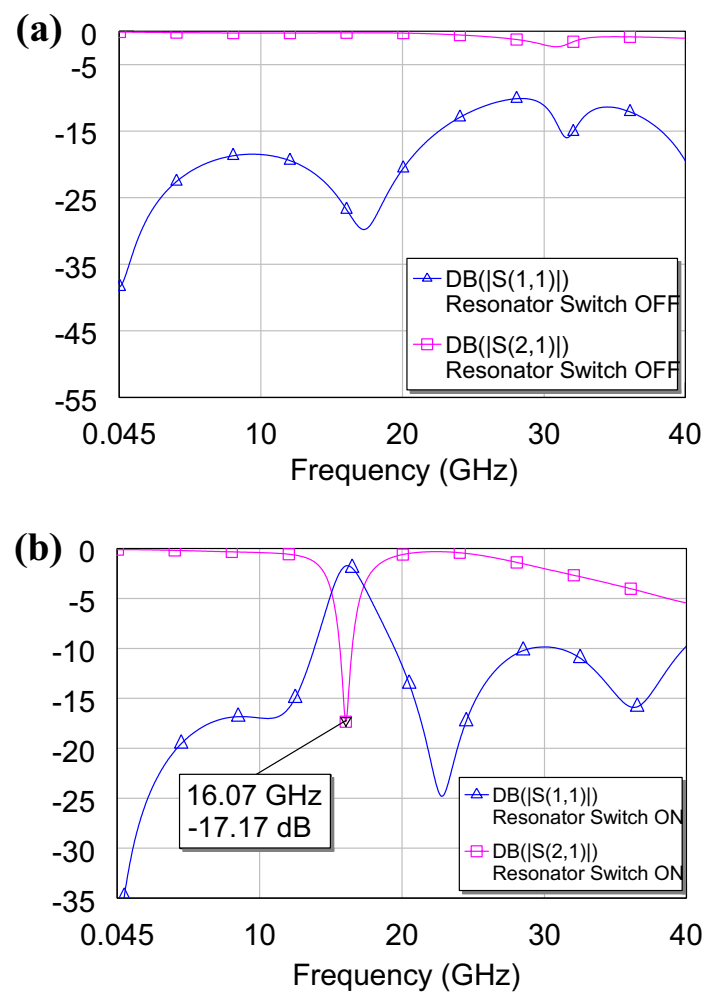

Fig. 2 Expected response of the triangular resonator on alumina in (a) the OFF state (cantilever UP) and b the ON state (cantilever DOWN), with $\mathrm{W}_{\mathrm{x}}=5.4 \mathrm{~mm}$ and $\mathrm{W}_{\mathrm{y}}=1.8 \mathrm{~mm}$

state). This is the first step before the actual manufacturing process that includes a cantilever to be really actuated by means of more than one standard lithographic process. Our work is just one mask process but it is an important demonstration about the possibility to manage the resonance properties of the triangles, even including a meta-material response. $2 \mathrm{D}$ simulations have been performed by means of the simulation software Applied Wave Research (AWR) Microwave Office. The expected response in terms of the scattering (S) parameters of the ON and OFF states of one of the exploited configurations on alumina are presented in Fig. 2, from where it is evident that the ratio between the two states is in the order of $17 \mathrm{~dB}$. Specifically, the size of the triangle used for the simulation, with reference to that presented in Fig. 1, is $\mathrm{W}_{\mathrm{x}}=5.4 \mathrm{~mm}$ and $\mathrm{W}_{\mathrm{y}}=1.8 \mathrm{~mm}$.

\section{Metamaterial properties of the proposed structure}

As discussed in the introduction, metamaterials (MMs) are artificial structures that can be designed to exhibit unusual electromagnetic properties, including the characteristic of simultaneous negative permittivity and permeability. The latter are more commonly referred to as left-handed materials (LHM) and their unique properties can be analyzed with a transmission line (TL) approach (Caloz and Itoh 2004). A potential advantage of the utilization of MMs is related, further the exotic applications like cloaking (Gabrielli and Poitras 2009), to the possibility of miniaturize high frequency structures (Martín et al. 2013; Simion 2013; Simion and Bartolucci 2014). This is an appealing property when microwave planar devices are considered (up to $30 \mathrm{GHz}$ ) in both microstrip and coplanar waveguide configurations (Simion et al. 2007). The general TL approach provides insight into the physical phenomena of LHMs and gives an efficient design tool for LH applications. LHMs can be included in a more general model of composite right/left handed (CRLH) structures, which also involve right-handed (RH) effects that occur naturally in practical LHMs (Simion et al. 2010). Actually, parasitic elements are always present in the real structures, thus imposing the necessity for a CRLH modeling. Starting with the Caloz and Itoh transmission line approach, we retrieved the $\varepsilon$ and $\mu$ parameters for the proposed configuration by means of the impedance $\mathrm{Z}$ and admittance $\mathrm{Y}$ parameters obtained by the Microwave Office simulations. The computation can also be performed starting from measured data, firstly converting the scattering $\mathbf{S}$ matrix into $\mathbf{Z}$ matrix as summarized in the following equations:

$Z_{11}=\frac{\left(\left(1+S_{11}\right)\left(1-S_{22}\right)+S_{12} S_{21}\right)}{\Delta} Z_{0}$

$Z_{21}=\frac{2 S_{21}}{\Delta} Z_{0}$ 
$Z_{22}=\frac{\left(\left(1-S_{11}\right)\left(1+S_{22}\right)+S_{12} S_{21}\right)}{\Delta} Z_{0}$

$\Delta=\left(\left(1-S_{11}\right)\left(1-S_{22}\right)-S_{12} S_{21}\right)$

where $Z_{i j}$ are the impedances and $S_{i j}$ the scattering parameters. As well established, the matrix representation allows for the evaluation of the electrical quantities on both ports of the equivalent network, being (11) and (22) the reflection indexes, whereas (21) and (12) refer to the transmission ones.

If we consider a two port symmetric and reciprocal network, a relationship between the $(\mathbf{Z}, \mathbf{Y})$ matrix electrical parameters, the dielectric scalar permittivity $\varepsilon$ and the magnetic scalar permeability $\mu$ can be obtained by means of the definition of the effective quantities $\mathrm{Z}_{\text {eff }}$ and $\mathrm{Y}_{\text {eff }}$ :

$Y_{\text {eff }}(\omega)=\frac{1}{Z_{21}(\omega)}$

$Z_{\text {eff }}(\omega)=2 \cdot\left(Z_{11}(\omega)-Z_{21}(\omega)\right)$

$\mu(\omega)=\frac{Z_{e f f}(\omega)}{i \cdot \omega}$

$\varepsilon(\omega)=\frac{Y_{e f f}(\omega)}{i \cdot \omega}$
Fig. 3 Real part of $\varepsilon$ and $\mu$ for the switch in the ON state. At resonance, when $\mathrm{f}=16.14 \mathrm{GHz}$, both values are negative

Fig. 4 Real part of $\varepsilon$ and $\mu$ for the switch in the OFF state. No resonance is obtained, because in this case the device is just a transmission line
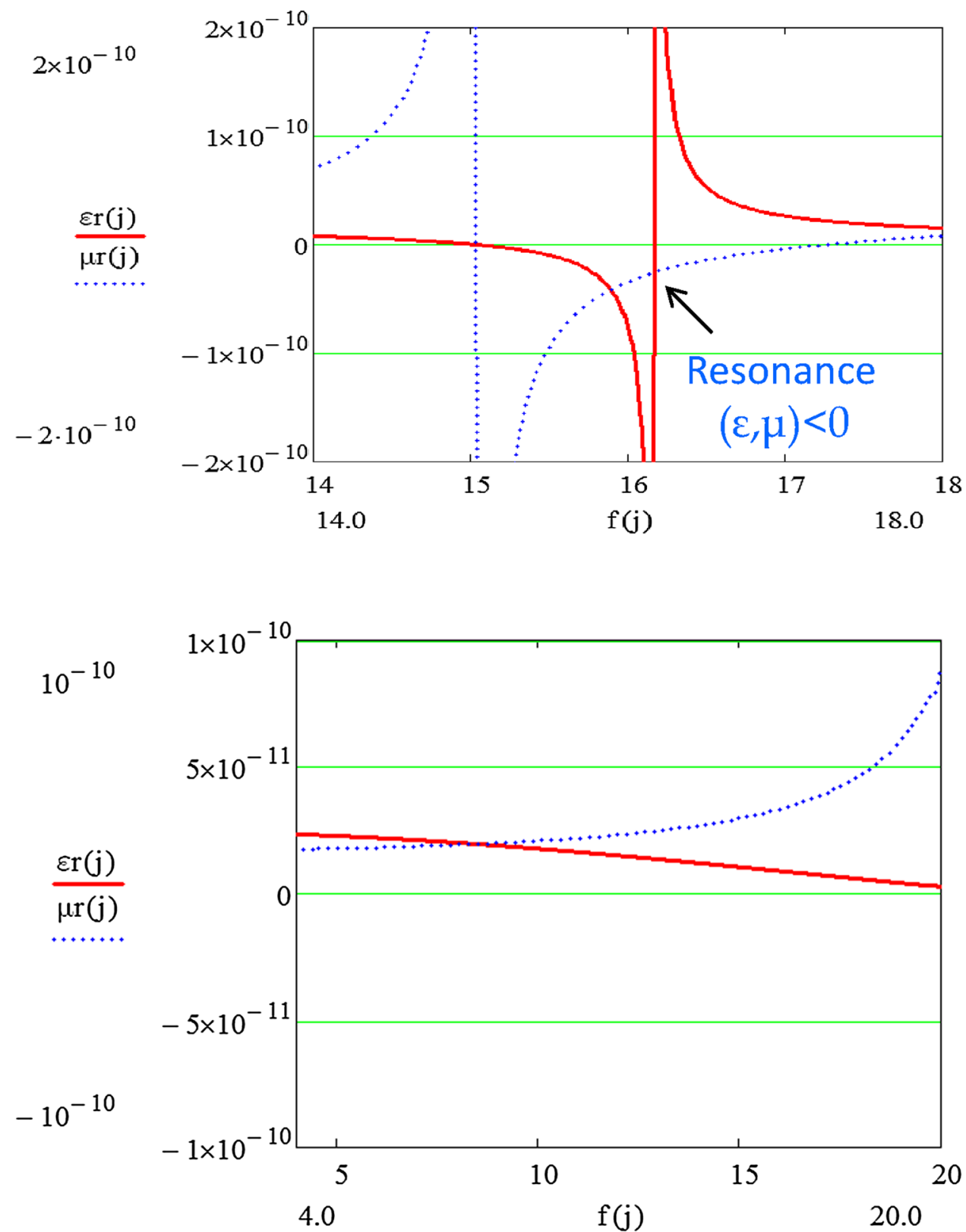
where $Z_{11}$ is the impedance measured at port 1 when current at port 2 is zero, $Z_{21}$ is the trans-impedance measured at port 2 when the current at port 2 is zero, and $\omega=2 \pi f$.

In Figs. 3 and 4 are plotted the effective values of the real part of $\varepsilon$ and $\mu$ computed by Eqs. (5), (6), (7) and (8) for the alumina structure in Fig. 1.

\section{Fabrication and test}

\subsection{Alumina substrate}

The metamaterial triangular split ring resonators have been produced by means of standard Clean Room processing set-up in the Institute for Microelectronic and Microsystems. The devices have been firstly manufactured on a 2-inch alumina substrate having a thickness of $635 \mu \mathrm{m}$. For the fabrication of the devices one lithography mask has been necessary and the details of the entire fabrication process are summarized as it follows:

1. Alumina substrate cleaning.

2. Physical Vapour Deposition of Chrome (100 ̊).

3. Physical Vapour Deposition of Gold (5000 $\AA$ ).

4. Spinning of $\mathrm{S} 18136000 \mathrm{rpm}$ for $120 \mathrm{~s}(1.1 \mu \mathrm{m})$.

5. Soft Bake on hot plate $\left(5 \mathrm{~min}\right.$ at $\left.90{ }^{\circ} \mathrm{C}\right)$.

6. UV Exposure (Devices Mask, $8 \mathrm{~s}$ of exposure to the UV Lights).

7. Photoresist Development (23 s in MF 319).

8. Wet etching of Gold.

9. Wet etching of Chrome.

10. Removal of residual photoresist (1165 Removal).

In the manufactured devices the ideal situation represented by a cantilever completely collapsed has been realized, to have a reference, ideal device matching as close as possible the theoretical design. For this purpose, no sacrificial layer has been used, but metal deposition has been performed directly onto the substrate. This configuration represents the $\mathrm{ON}$ state of an electrostatic actuated metal cantilever beam. The OFF configuration has been emulated by the absence of the metal cantilever beam (Fig. 5, on the left upper corner of the structure).

Actually, as available in current literature, a $3 \mu \mathrm{m}$ gap is enough to provide a good isolation for the entire device when the bridge is in the UP position. This preliminary work has been performed years ago also by removing a bridge belonging to a CPW shunt device, to demonstrate, crossing simulation and experimental data, that a negligible influence is obtained on the microwave performances. Actually, a bridge suspended on a CPW line does not involve additional losses, exception done for not optimized discontinuities related to its accommodation in the place where it is manufactured.

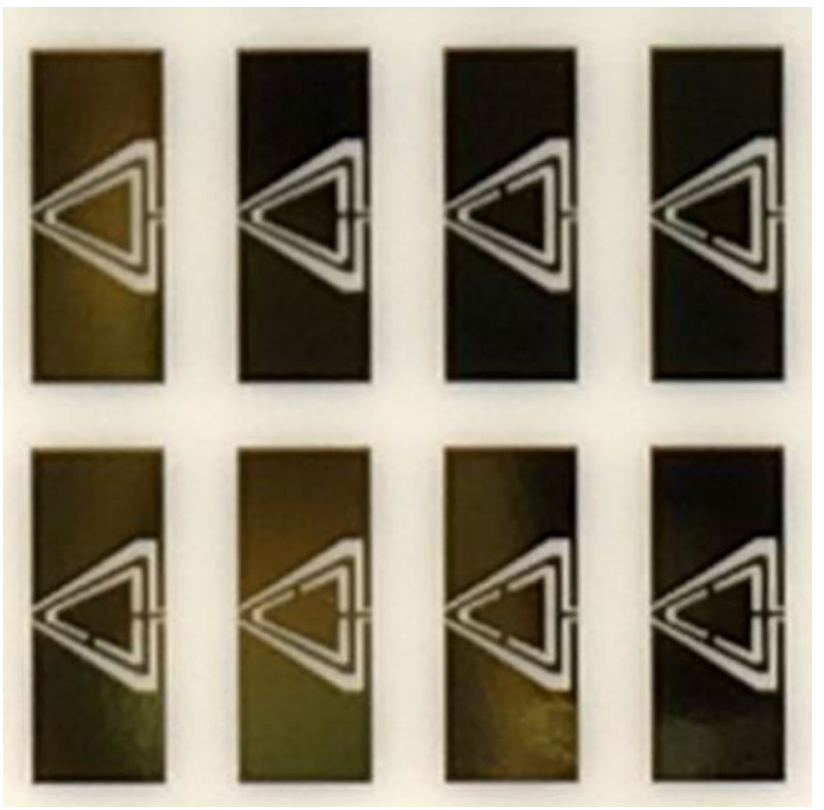

Fig. 5 Single cell of the manufactured devices. The discontinuities have been inserted in all the three arms of the triangle to investigate the possibility to have a frequency tuning by RF MEMS switches actuated around the inner triangle

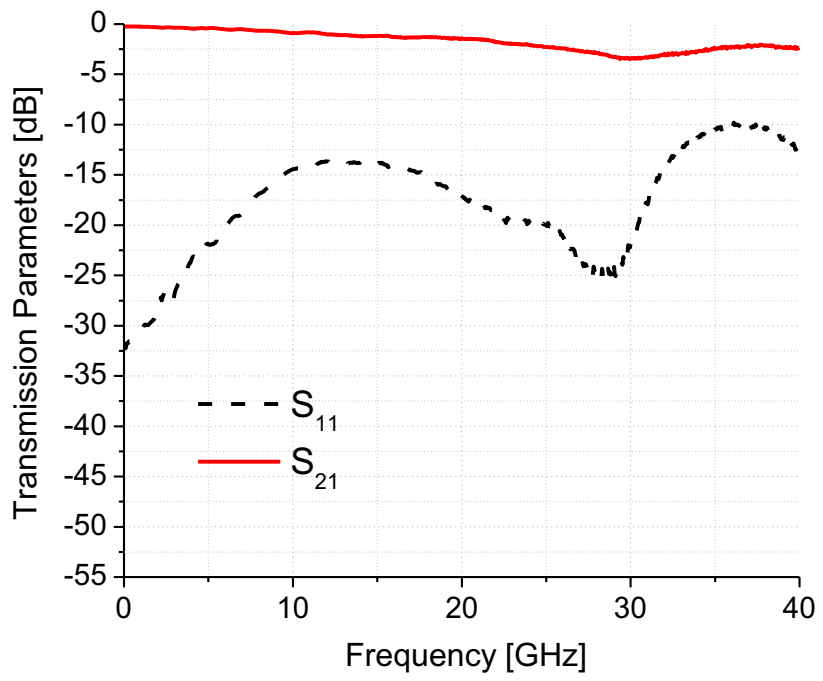

Fig. 6 S-parameters measurement of the triangular CPW when cantilever switch is in the OFF state (bridge UP)

Concerning the bridge in the DOWN position, i.e. the configuration for having a technologically actuated device to have a prediction of the best obtainable performance, the metal-to-metal contact is not influenced by the shape inhomogeneities due to the actual fabrication process. Anyway, looking to past experimental findings, a contribution lower than $0.1 \mathrm{~dB}$ is expected by the real situation. 
RF tests on the MM resonators have been performed and they are presented in Figs. 6 and 7. In Fig. 8 the device under test is shown. The measured electrical performances are in good agreement with the performed simulations presented before. In particular, the devices have been designed to have resonance peak (in the ON State) around $16 \mathrm{GHz}$.

Triangles manufactured on alumina and having double size with respect to the previous ones have been also tested, giving, as a scale factor, an operating resonance frequency around $8 \mathrm{GHz}$, as it is shown in the following Fig. 9.

To demonstrate the tuning property of the triangular geometry, the frequency jump to X-band is evidenced in Fig. 10 where three RF MEMS switches have been actuated around the inner triangle, which has the same size as before. The number of discontinuities introduced by the presence of

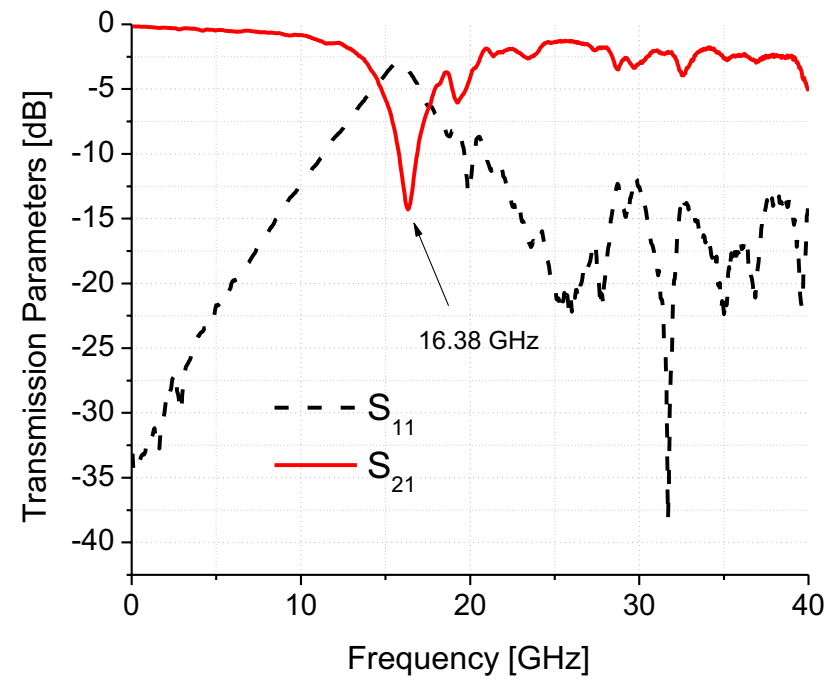

Fig. 7 S-parameters measurement of the triangular resonator when cantilever switch is in the ON state (bridge DOWN, actuated)
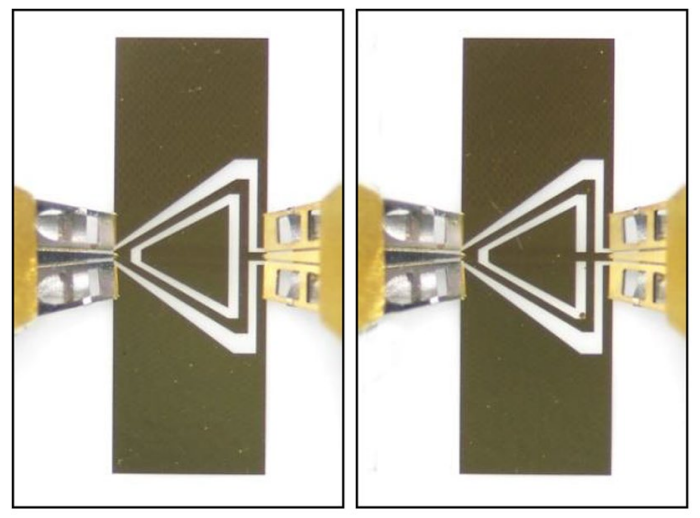

Fig. 8 Picture of the tested devices realized on alumina (left side: OFF state, right side: ON State)

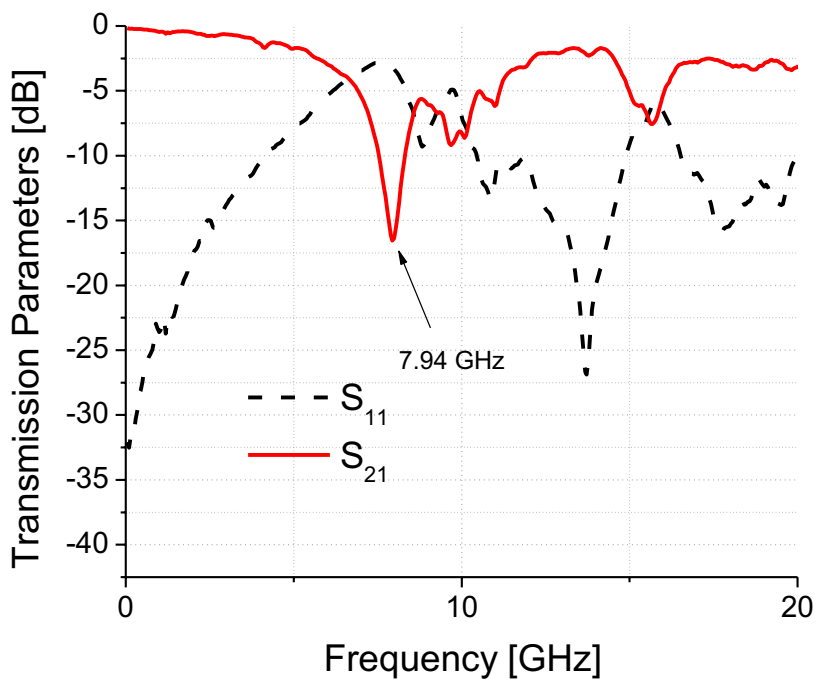

Fig. 9 Response of a triangular device having double size $(3.6 \times 5 \mathrm{~mm})$ with respect to Fig. 8. A rough scale-down of a factor two is obtained for the frequency of resonance

the actuated switches allows for digital tunability up to $20 \%$ with respect to the frequency of resonance given in Fig. 9.

\subsection{Silicon substrate}

Once the proof of concept has been demonstrated, we realized that the technological steps in the cantilever beam manufacturing process must be more than four. By decreasing the frequencies (X-band for example) and by increasing the dimensions of the triangular resonator, the cantilever used as a switch maintains its geometry and size. Actually, it is hard to have a good MEMS device on alumina, due to the material intrinsic roughness, which renders difficult to have a reliable electro-mechanical response when the metal beam is collapsed.

Although alumina is well known since decades in the microwave applications, and the improvement of the RF MEMS technology on this kind of substrate could help in obtaining configurations suitable of a better power handling, we changed the substrate to realize the cantilever beam working at higher frequencies for the above outlined technological reasons. Then, we used for the $16 \mathrm{GHz}$ device a silicon substrate, which is extremely smooth and flat and dimensionally stable, allowing fine line lithography. It can provide a better integration using a more mature technology for reliable RF MEMS configurations.

A study of the cantilever beam has been ultimated before the integration between switch and resonator to investigate the mechanical behavior of the beam (Fig. 11a), the actuation voltage (Fig. 11b) and the contact resistance (Fig. 11c).

We verified that the initial actuation voltage is $84 \mathrm{~V}$, value that starts decreasing with mechanical cycling with an 
Fig. 10 Microwave response of the double size device on alumina with three switches actuated. The device configuration under test is given on the left
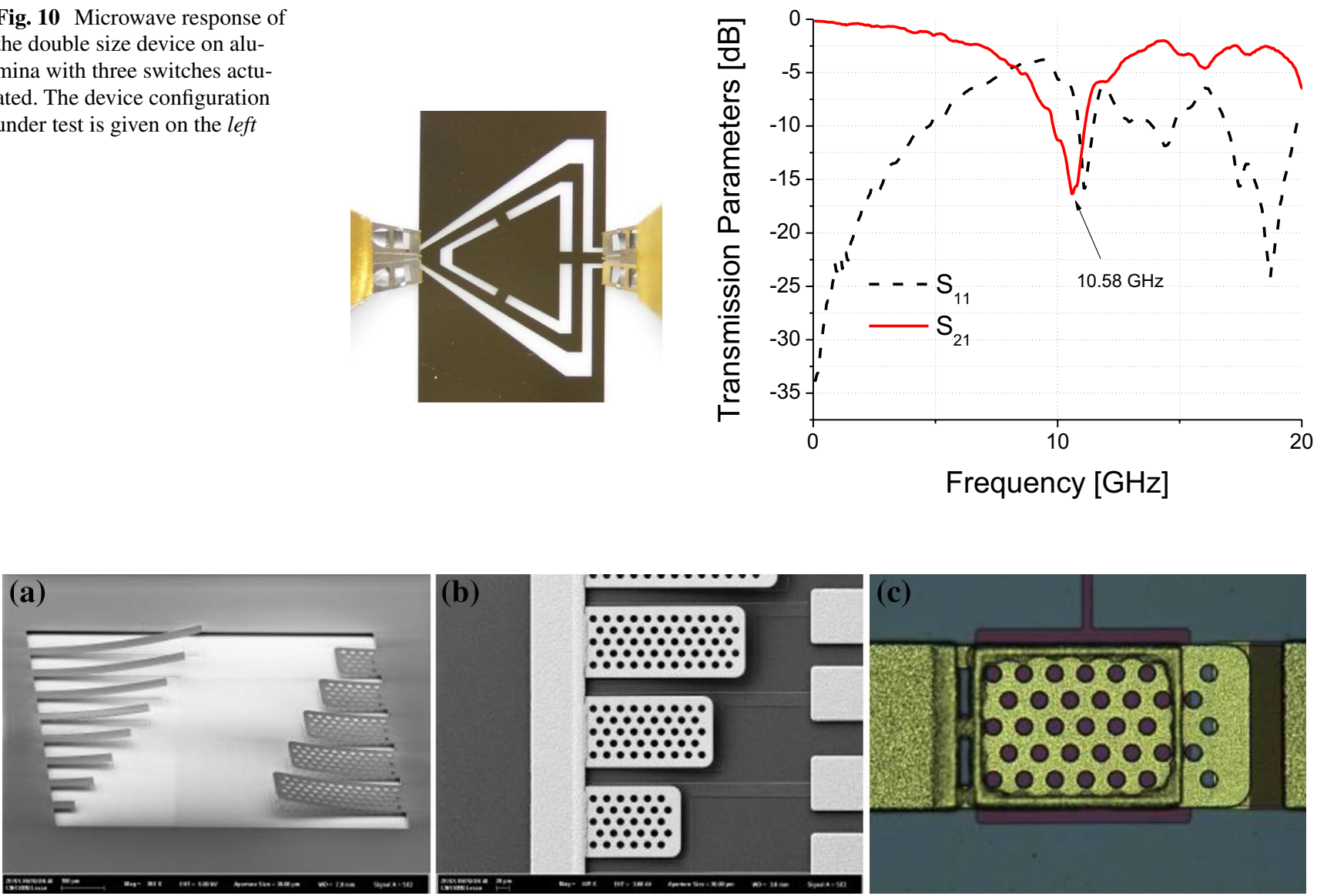

Fig. 11 Cantilever beam manufactured on silicon wafer. The process has been set up to avoid mechanical deflection of the cantilever, like in (a), properly choosing the length, width and material parameters.

asymptotic trend to $60 \mathrm{~V}$ after $10^{6}$ cycles. In Fig. 12a, b are summarized the measured RF performances in terms of the Return Loss $\left(\mathrm{S}_{11}\right)$ and Transmission $\left(\mathrm{S}_{21}\right)$ when the bridge is in the DOWN state, i.e. when the beam collapsed because of the applied actuation voltage. Cycles with a period $\mathrm{T}=2 \mathrm{~ms}$ have been used, with a duty cycle $\mathrm{D}=50 \%$. It can be observed that very good electrical performances are obtained, stable with respect to the mechanical stress.

To manufacture the RF MEMS switches, a standard 4-inch diameter $(10 \mathrm{~mm})$ High Resistivity P-type Silicon wafer from TopSil Semiconductor Material has been used: its nominal thickness is $525 \mu \mathrm{m}$ and its nominal surface resistivity $\rho$ is $10 \mathrm{k} \Omega \cdot \mathrm{cm}$. The input/output lines on silicon for the RF signal have been changed with respect to those designed on alumina for improving the electrical matching of the device, based on the preliminary experimental verifications. Moreover, to be as close as possible to the next realization we have designed the collapsed bridge accounting also for the holes useful in the surface micromachining processing to eliminate the sacrificial layer during the etching.
Actuation pads for imposing the actuation voltage have been realized in (b). A detail of the actuation area during the contact resistance measurement is shown in (c)

In the following, theoretical and experimental results for the triangle characterized by double-size geometry for the triangular resonator will be shown, with the frequency of resonance around $8 \mathrm{GHz}$. The overall dimensions of the devices are $\mathrm{W}_{\mathrm{x}}=10 \mathrm{~mm}$ and $\mathrm{W}_{\mathrm{y}}=4.5 \mathrm{~mm}$.

The realization process follows the same technological steps previously described for the alumina substrate, as mainly photolithographic steps suitable to be performed on any surface have been used. From the point of view of the expected electromagnetic performances, we do not foresee any substantial variation between the utilization of Alumina or Silicon, exception done for the already discussed improved reliability for the switch, due to an easiest fabrication process and to a better contact resistance on the flat silicon surface. The two materials exhibit similar dielectric properties, and the slightly different thickness of the Silicon substrate plays at this stage a marginal role in the electrical response of the device also by using the same mask. Also in this second design, measurements on manufactured devices match in a satisfactory way the theoretical predictions. In Fig. 13, the manufactured structures are shown, 

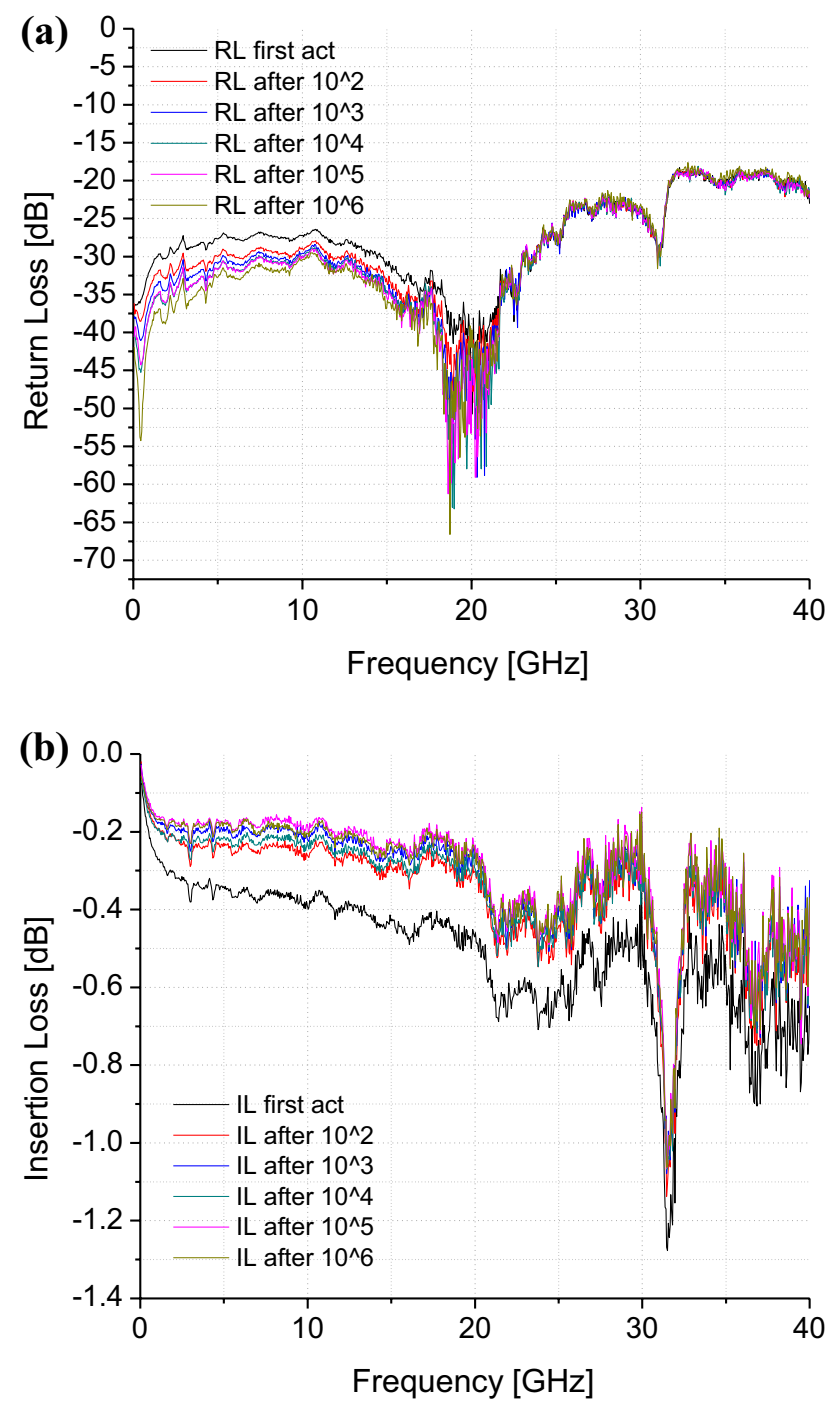

Fig. 12 Behavior of the MEMS in DOWN state (actuated) in terms of mechanical cycling, up to $10^{6}$ actuations, by imposing a period of $2 \mathrm{~ms}$ and a duty cycle of $50 \%$ : a the Return Loss $\left(\mathrm{S}_{11}\right.$, or reflection measurement) is well below $-20 \mathrm{~dB}$ up to $30 \mathrm{GHz}$, and $\mathbf{b}$ the Insertion Loss $\left(\mathrm{S}_{21}\right.$, or transmission measurement) is in the order of $-0.5 \mathrm{~dB}$ up to $30 \mathrm{GHz}$

with evidence for the reference one without switches (a), for those with technologically actuated switches on lateral sides (b)-(d) and for the detail of the collapsed switch (e).

In the following Figs. 14, 15, 16 and 17, we plotted the microwave responses of the devices shown in Fig. 13, together with the theoretical response obtained by means of the Microwave Office software planar electromagnetic simulations (bi-dimensional, or 2D structures).

From the analysis of the experimental results, it turns out that a wide frequency tuning can be provided in the case of the silicon-based devices, including frequencies spanning between 7 and $15 \mathrm{GHz}$. Nevertheless, the position of the switch, acting as a discontinuity of the resonating structure, needs an optimization in the position around the inner triangle to provide a peak depth with comparable performances over the entire band.

For a useful comparison, some realization of resonators in CPW are described in (Safwat et al. 2006) and (Lim et al. 2002); in both cases the filtering function is obtained by stub loading the ground planes of the CPW. Regarding the effective electrical dimensions, the geometry presented in (Safwat et al. 2006) is $\mathrm{W}_{\mathrm{x}}=0.14 \lambda_{\mathrm{g}} \times \mathrm{W}_{\mathrm{y}}=0.49 \lambda_{\mathrm{g}}$ and our design (referring to results in Fig. 15 and geometry in Fig. 13b is $\mathrm{W}_{\mathrm{x}}=0.23 \lambda_{\mathrm{g}} \times \mathrm{W}_{\mathrm{y}}=0.31 \lambda_{\mathrm{g}}$, where $\lambda_{\mathrm{g}}$ is the guided wavelength computed at the resonance frequency.

\section{The equivalent circuit}

The development of effective models of passive microwave components to be implemented in CAD programs plays a crucial role for the accurate simulations of such structures. To this end circuit topologies composed by resonant LC elements are typically adopted (Giannini et al. 1992; Muldavin and Rebeiz 2000; Bartolucci et al. 1995; Rizk et al. 2001). In this section the configuration shown in Fig. 18 is proposed for the modeling of the triangular resonator on the Alumina substrate (considering the cantilever switch in the ON state). The choice of this topology is strictly related with the frequency behavior of $\left|S_{21}\right|$ plotted in Fig. 19. In particular, the shunt connected circuit has been designed to resonate at the frequency of the band stop peak $(16.38 \mathrm{GHz})$, so having a mathematical condition to be fulfilled by $\mathrm{L}$ and $\mathrm{C}$. The impedance $\mathrm{Z}$ of this circuit can be written as:

$Z=R+j \omega L+\frac{1}{j \omega C}=R+j X$

being:

$X=\frac{L}{\omega}\left(\omega^{2}-\omega_{p}^{2}\right), \quad \omega_{p}=2 \pi f_{p}=\frac{1}{\sqrt{L C}}, \quad f_{p}=16.38 \mathrm{GHz}$

The equivalent circuit in Fig. 18 is a two-port network, and can be therefore characterized by means of the scattering parameters.

For $S_{21}$ the following expression can be obtained:

$S_{21}=\frac{2}{2+\frac{Z_{0}}{Z}}=\frac{1}{1+Z_{0} \frac{R-j X}{R^{2}+X^{2}} 0.5}$

For $\omega=\omega_{p}$ Eqs. (9) and (10) give:

$\left|S_{21}\right|_{f=f_{p}}=\frac{1}{1+\frac{Z_{0}}{R} 0.5}$ 

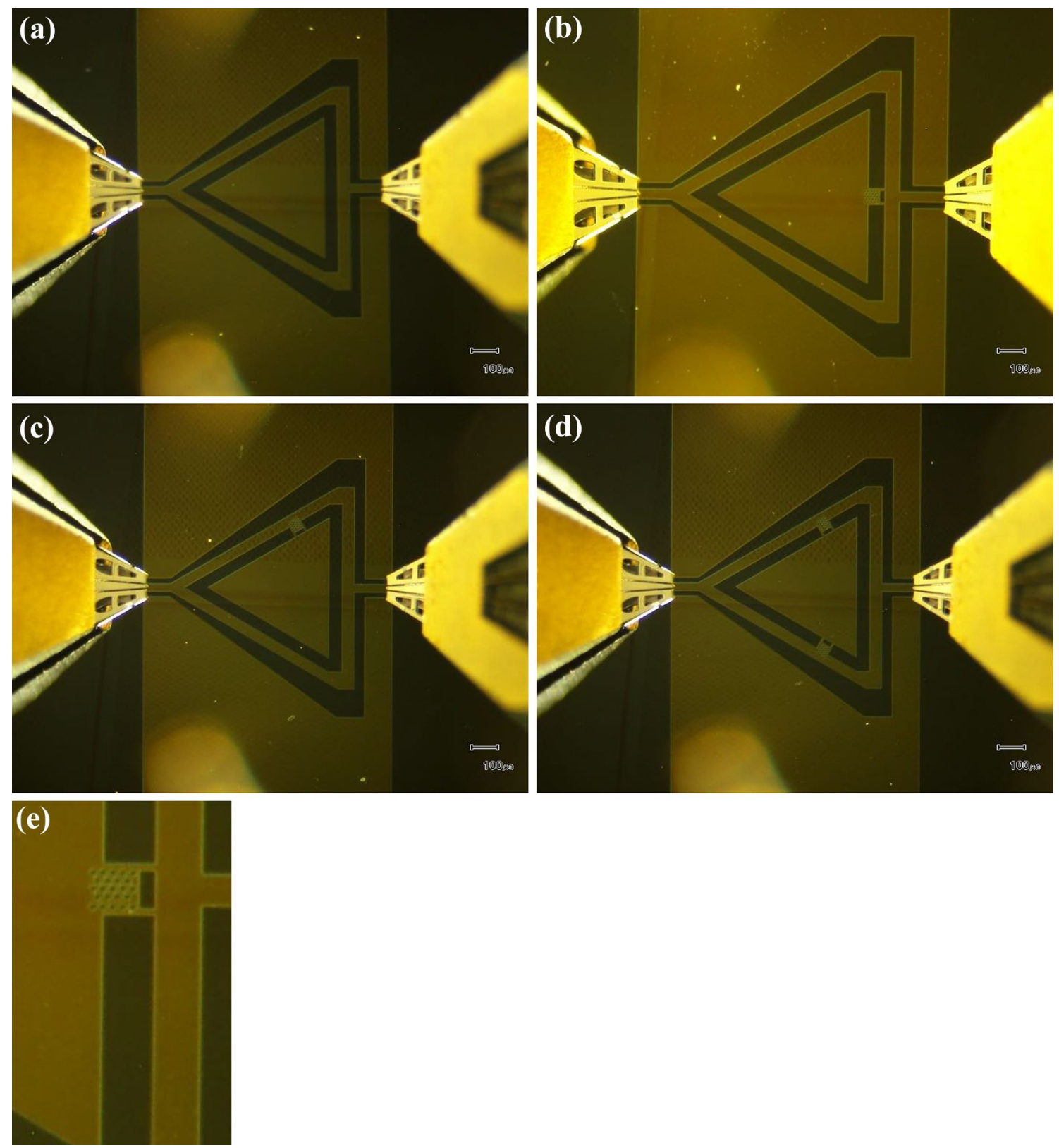

Fig. 13 Triangular resonators manufactured on silicon: a reference line with no switches, $\mathbf{b}$ resonator with one switch along the same line of the $\mathrm{CPW}, \mathbf{c}$ resonator with one switch on the side, and $\mathbf{d}$ with

and

$R=\frac{0.5 Z_{0}\left|S_{21}\right|_{f=f_{p}}}{1-\left|S_{21}\right|_{f=f_{p}}}$

The value of $\left|S_{21}\right|$ to be used in (13) is computed by the measured result at $f_{p}$, that is in our case $\left|S_{21}\right|_{d B}=-14 \mathrm{~dB}$, so obtaining $\left|\mathrm{S}_{21}\right|_{\mathrm{f}=\mathrm{fp}}=0.2$. Inserting this value in (6) we find: $R=6 \Omega$. The complete characterization of the equivalent circuit requires the evaluation of the reactive elements. To this switches on both lateral sides. In e the detailed view of the technologically actuated RF MEMS switch is shown

end the following procedure is used. For a fixed frequency $f_{1}$ the $\left|S_{21}\right|$ provided by (4) must be equal to the measured $\left|S_{21}\right|$ at $f_{1}\left(\left|\mathrm{~S}_{21}\right|_{\text {meas }}\right)$. Imposing this condition the inductance $\mathrm{L}$ is computed in terms of $\left|S_{21}\right|_{\text {meas }}$. More in detail, an auxiliary unknown $u$ and an auxiliary variable $q$ can be defined as:

$$
\begin{aligned}
& u=\frac{L^{2}}{\omega_{1}^{2}}\left(\omega_{1}^{2}-\omega_{p}^{2}\right)^{2} \\
& q=\frac{4}{\left|S_{21}\right|_{\text {meas }}^{2}}-4
\end{aligned}
$$



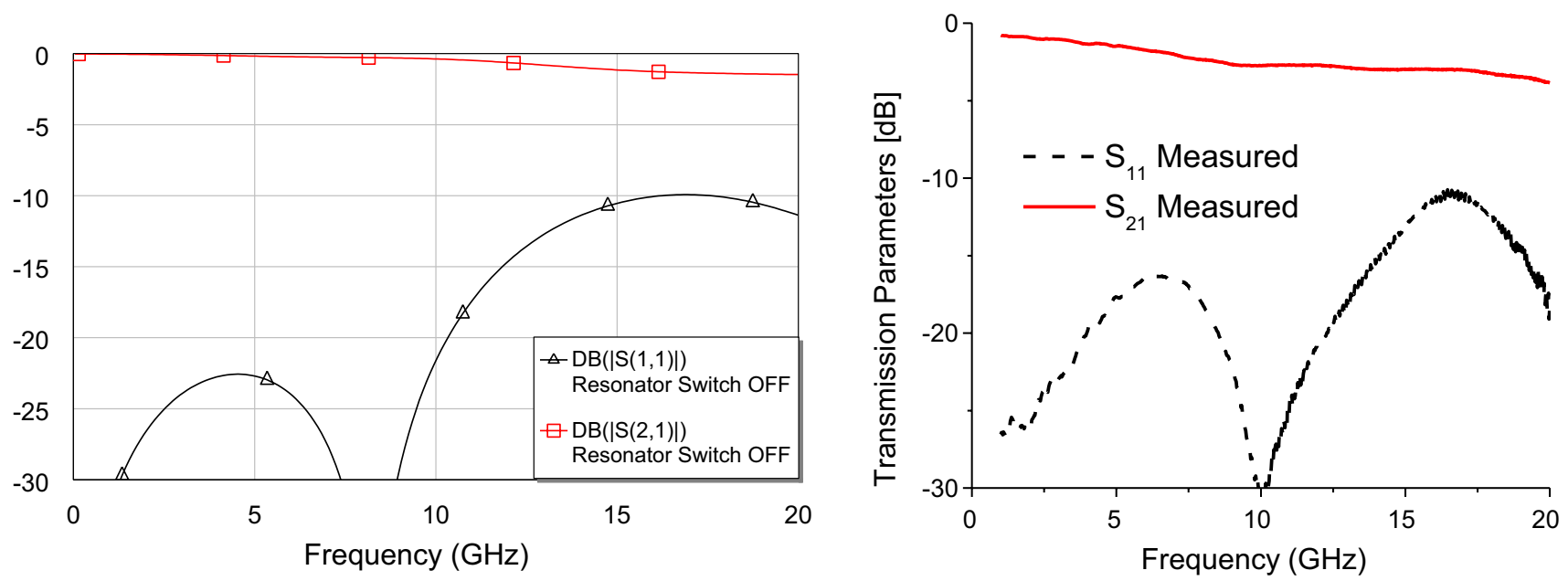

Fig. 14 Reference line response. Simulation (left) and experimental measurement (right), with respect to geometry Fig. 13a, "OFF" state

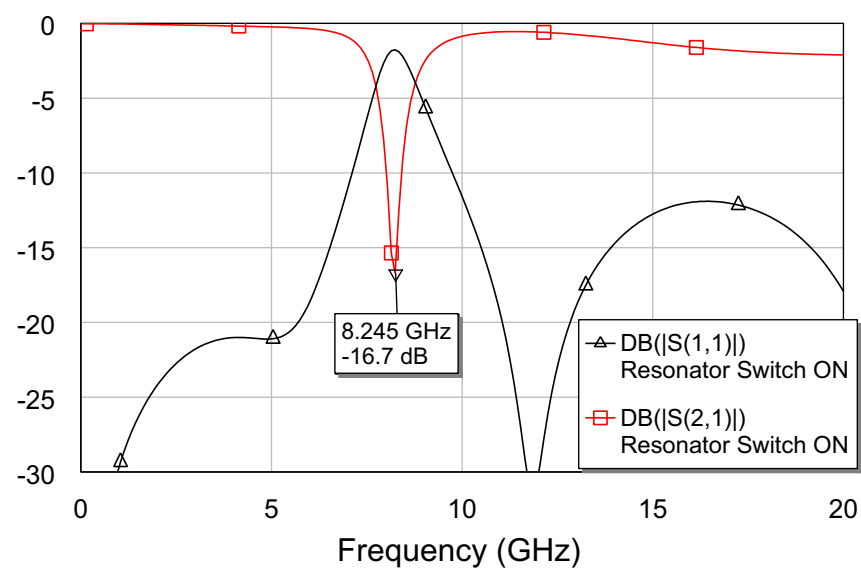

Fig. 15 Simulation (left) and experimental response for the device in Fig. 13b (one switch actuated). In this case, the significant shift between the predicted frequency of resonance and the measured one

being $\omega_{1}=2 \pi f_{1}$. After some algebra the following quadratic equation is obtained:

$u^{2}+u\left[2 R^{2}-\left(Z_{0}+4 R\right) \frac{Z_{0}}{q}\right]+R^{4}-\frac{Z_{0}}{q} R^{2}\left(Z_{0}+4 R\right)=0$

The solutions of (15) can be written as:

$u_{1}=-R^{2}+\left(Z_{0}+4 R\right) \frac{Z_{0}}{q}$

$u_{2}=-R^{2}$

Since $L$ must be a real number only $u_{l}$ can be chosen. From the above equations we have:

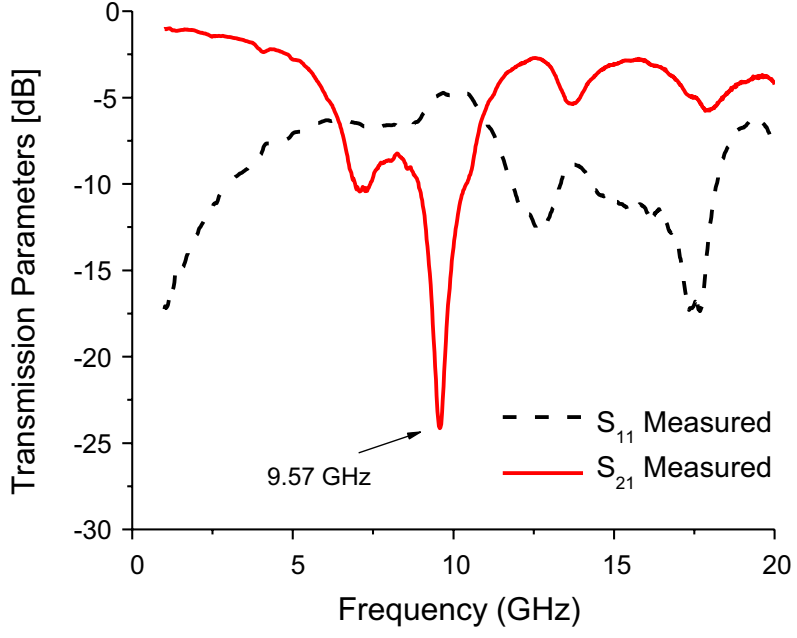

is probably due to the presence of the spurious, unwanted resonance close to the main one

$L=\frac{\omega_{1}}{\omega_{1}^{2}-\omega_{p}^{2}} \sqrt{-R^{2}+\left(\frac{Z_{0}}{4}+R\right) \frac{Z_{0}\left|S_{21}\right|_{f_{1}-\text { meas }}^{2}}{1-\left|S_{21}\right|_{f_{1}-\text { meas }}^{2}}}$

According to the definition of $\omega_{\mathrm{p}}$ the capacitance $\mathrm{C}$ is computed as follows:

$C=\frac{1}{L \omega_{p}^{2}}$

The chosen $f_{1}$ is $20 \mathrm{GHz}$, and the corresponding value for $\left|S_{21}\right|$ is 0.71 . Inserting these values in (17) and (18) we find $\mathrm{L}=0.715 \mathrm{nH}, \mathrm{C}=0.132 \mathrm{pF}$. The circuit model depicted in Fig. 18 is implemented in the software package 


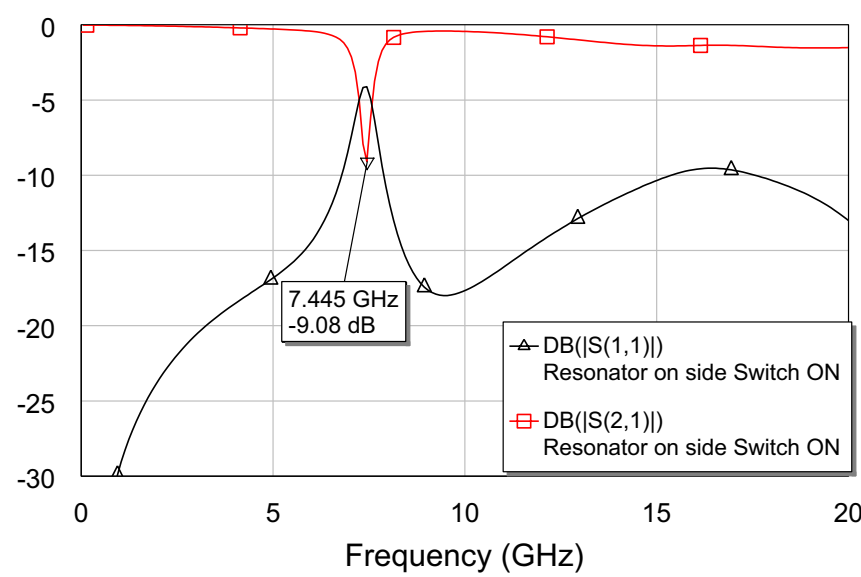

Fig. 16 Simulation (left) and experiment (right) for the resonator in Fig. 13c (actuated switch on one side). This configuration, looking to the depth of the peak, appears not optimized, and probably the posi-

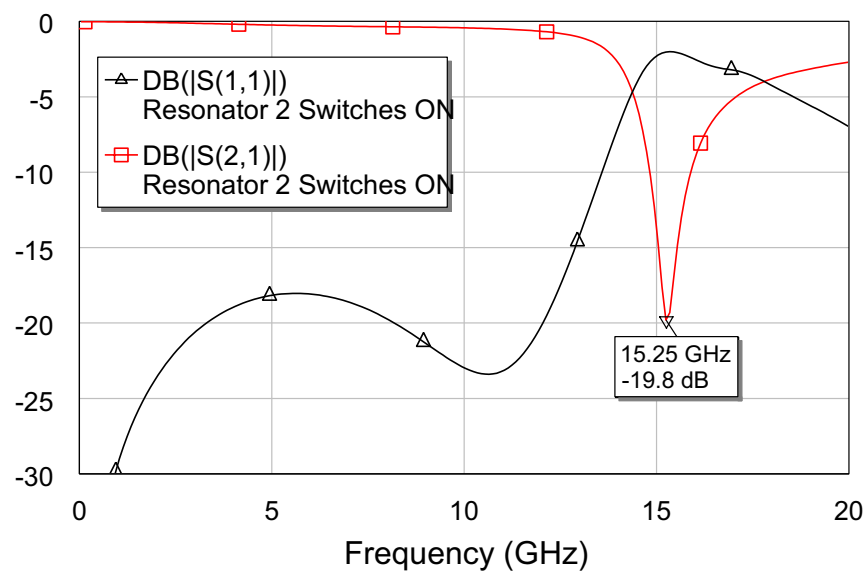

Fig. 17 Expected response (left) and experimental verification (right) for the configuration in Fig. 13d (actuated switches on both sides of the triangle). Also in this case the expected behavior is more perfor-

AWR Microwave Office (MWO), by using for the R, L, C elements the above achieved numerical data. The simulated behavior of $\left|S_{21}\right|$ versus frequency is plotted in Fig. 19, in agreement with the measured results presented in Fig. 7.

\section{Conclusion}

In this paper a triangular resonator exhibiting metamaterial properties has been studied, with possible narrow band tunable filtering applications, on both alumina and silicon substrates. In particular, a triangular CPW

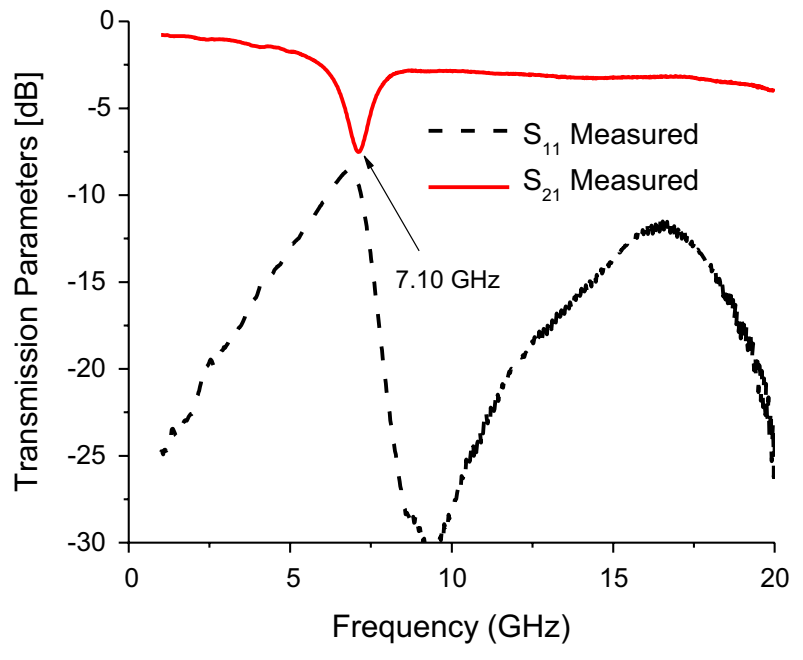

tion of the switch is mainly responsible for the non-optimal electrical response of the device

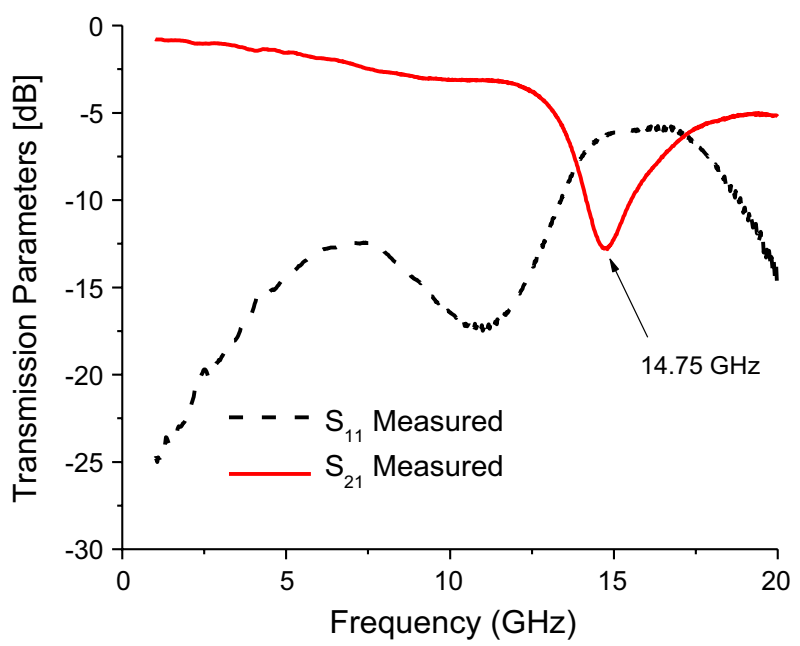

mant with respect to the experiment, with a $-20 \mathrm{~dB}$ peak in comparison with the measured value of $-13 \mathrm{~dB}$

configuration has been designed, manufactured and tested up to $40 \mathrm{GHz}$, including a technologically actuated cantilever beam. The behavior of the structures has been studied with the switch in the ON and OFF state evidencing a resonance only in the ON state, i.e. when the switches are actuated and thus creating the split. The effective dielectric constant and magnetic permeability have been retrieved by means of the equivalent transmission line model, showing a meta-material response within the resonance band, potentially useful for miniaturization purposes. An equivalent circuit for emulating the structure has been obtained and validated by means of a circuital 


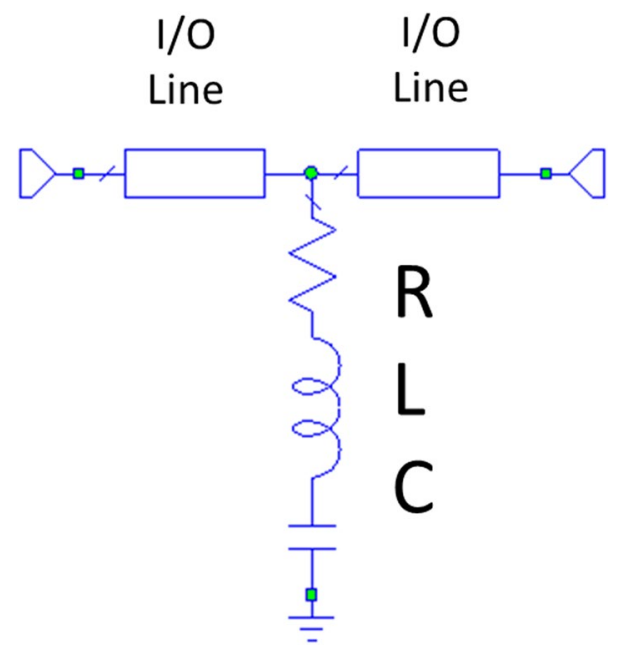

Fig. 18 The proposed equivalent circuit. As it is shown, a quite simple approach is enough for emulating the resonating structure with the cantilever switch is in the ON state. In the present case, after the input/output (I/O) lines we have the RLC circuit, where $\mathrm{C}=0.132$ $\mathrm{pF}, \mathrm{L}=0.715 \mathrm{nH}$ and $\mathrm{R}=6 \mathrm{ohm}$ have been inferred from the model

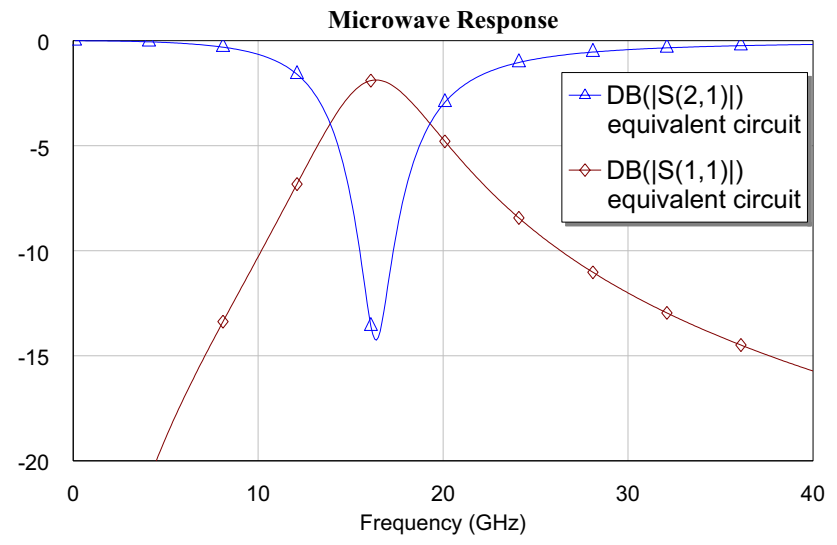

Fig. 19 Reflection $\left|S_{11}\right|$ and transmission $\left|S_{21}\right|$ versus frequency provided by the equivalent circuit

simulator, by means of the analytic expressions for the computation of the lumped elements presented in this model. Since the tests clearly exhibited a good agreement with simulations, and taking into account the state-of-art for the RF MEMS manufacturing, a run is presently ongoing to realize the fully operational device on silicon, including the cantilever switch to be electro-statically actuated, taking advantage from the current process reliability of the MEMS silicon technology.

\section{References}

Bartolucci G, Giannini F, Limiti E, Marsh SP (1995) MIM capacitor modeling: a planar approach. IEEE Trans Microw Theory Tech 43(4):901-903
Caloz C, Itoh T (2004) Transmission line approach of left-handed (LH) structures and microstrip implementation of an artificial LH transmission line. IEEE Trans Antennas Propag 52(5):1159-1166

Engheta N, Ziolkowski RW (2006) Metamaterials: physics and engineering explorations. Wiley, New Jersey

Gabrielli JC, Poitras ML (2009) Silicon nanostructure cloak operating at optical frequencies. In: Nature photonics advanced online publication, vol 3 (8), pp 461-463

Giannini F, Bartolucci G, Ruggieri M (1992) Equivalent circuit models for computer aided design of microstrip rectangular structures. IEEE Trans Microw Theory Tech 40(2):378-388

Hong J-S, Li S (2004) Theory and experiment of dual-mode microstrip triangular patch resonators and filters. IEEE Trans Microw Theory Tech 52(4):1237-1243

Jalali M, Sedghi T, Zehforoosh Y (2009a) Miniaturization of waveguides dual band antenna using TSRR-WS metamaterials. Int J Computer Electr Eng 1(5):624-626

Jalali M, Sedghi T, Shokri M (2009b) A novel metamaterial SRR for waveguide antenna. In: Proceedings of Mediterranean Microwave Symposium (MMS), Tangiers, Morocco

Krupka J, Breeze J, Centeno A, Alford N, Claussen T, Jensen L (2006) Measurements of permittivity, dielectric loss tangent, and resistivity of float-zone silicon at microwave frequencies. IEEE Trans Microw Theory Tech 54(11):3995-4001

Lim J-S, Kim C-S, Lee Y-T, Ahn D, Nam S (2002) A spiral-shaped defected ground structure for coplanar waveguide. IEEE Microw Wirel Compon Lett 12(9):330-332

Martín F, Falcone F, Bonache J, Marqués R, Sorolla M (2013) Miniaturized coplanar waveguide stop band filters based on multiple tuned split ring resonators. IEEE Microw Wirel Compon Lett 13(12):511-513

Muldavin JB, Rebeiz GM (2000) High-isolation CPW MEMS shunt switches-part 1: modeling. IEEE Trans Microw Theory Tech 48(6): 1045-1052

Rizk J, Tan GL, Muldavin JB, Rebeiz GM (2001) High-isolation W-band MEMS switches. IEEE Microw Wirel Compon Lett 11(1):10-12

Sabah C (2008) Analysis, applications, and a novel design of double negative metamaterials. PhD Thesis, University of Gaziantep, Gaziantep, Turkey

Sabah C, Uckun S (2008) Triangular split ring resonator and wire strip to form new metamaterial. In: Proceedings of XXIX General Assembly of the International Union of Radio Science, Chicago, Illinois, USA

Sabah C, Cakmak AO, Ozbay E, Uckun S (2010) Transmission measurement of a new metamaterial sample with negative refraction index. Physica B 405:2955-2958

Safwat A, Podevin F, Ferrari P, Vilcot A (2006) Tunable bandstop defected ground structure resonator using reconfigurable dumbbell-shaped coplanar waveguide. IEEE Trans Microw Theory Tech 54(9):3559-3564

Simion S (2013) Design method of miniaturized ring coupler using phase shifters consisting of fully disturbed composite right/lefthanded structures. Prog Electromagn Res B 50:61-76

Simion S, Bartolucci G (2014) Broadband and small-size $3 \mathrm{~dB}$ ring coupler. Prog Electromagn Res Lett 44:23-28

Simion S, Marcelli R, Sajin G (2007) Small size CPW silicon resonating antenna based on transmission line meta-material approach. IEE Electron Lett 43(17):908-909

Simion S, Marcelli R, Bartolucci G, Craciunoiu F, Lucibello A, De Angelis G, Muller A, Bunea AC, Sajin GI (2010) Composite Right/Left Handed (CRLH) based devices for microwave applications. Chapter 6. In: Mukherjee M (ed.) Advanced Microwave and Millimeter Wave Technologies Semiconductor Devices Circuits and Systems; chapter 6. IN-TECH Publisher, pp 89-112. doi:10.5772/8742. http://www.intechopen.com 
Vidyalakshmi MR, Raghavan S (2009) A CAD model of triangular split ring resonator based on equivalent circuit approach. In: Proceedings of Applied Electromagnetics Conference (AEMC), Kolkata, India

Wolf I (2006) Coplanar microwave integrated circuits. Wiley, New Jersey

Xiao J-K (2012) Novel microstrip dual-mode bandpass filter using isosceles triangular patch resonator with fractal-shaped structure. J Electromagn Wave Appl 21(10):1341-1351
Zhu C, Liang CH, Chen L (2008) A novel left-handed material composed of triangular open-loop resonators. Proceedings of International Workshop on Metamaterials. Nanjing, China, pp 188-190

Zhu C, Ma JJ, Chen L, Liang CH (2009) Negative index metamaterials composed of triangular open-loop resonator and wire structures. Microw Optical Technol Lett 51(9):2022-2025 